\title{
Pharmacological Class of Investigational Therapy
}

National Cancer Institute

\section{Source}

National Cancer Institute. Pharmacological Class of Investigational Therapy. NCI

Thesaurus. Code C98768.

The drug class of the therapy being investig ated in the study or trial. 